\title{
Teleophthalmology consultations-how do we keep our patients safe?
}

\author{
Matthew Gillam (i) ${ }^{1} \cdot$ Ji-Peng Olivia Li $\mathbb{1}^{1}{ }^{1}$ - Caroline L. S. Kilduff ${ }^{1} \cdot$ Rishi Ramessur $^{1} \cdot$ Alice A. P. Thomas $^{1}$ • \\ Abison Logeswaran ${ }^{1} \cdot$ Badrul Hussain $^{1}$ - Peter B. M. Thomas ${ }^{2} \cdot$ Gordon Hay $^{1,3}$
}

Received: 28 September 2020 / Revised: 8 October 2020 / Accepted: 12 October 2020 / Published online: 26 October 2020

(c) The Royal College of Ophthalmologists 2020

Covid-19 has brought the use of remote ophthalmic consultations (teleophthalmology) to the forefront of ophthalmic services in a way that was completely unexpected prior to the pandemic. Recent publications have evaluated the ability of teleconsultations to determine ophthalmic diagnoses and plan treatment [1]. They have also shown that patients are satisfied with teleophthalmology as a means of accessing eye care [2]. The most important consideration, when using teleophthalmology, is to manage patients safely. After all, every physician has sworn to 'first, do no harm' and this applies equally to Ancient Greek consultation methods as it does to those used in the 21st century.

The Moorfields Accident and Emergency teleophthalmology service was rapidly rolled out to complement our ongoing face-to-face emergency department service. Patient safety was the focus from the start and we developed guidelines and policies to maximise this, in keeping with the GMC key principles for remote consultations and prescribing, the first of which is 'make patient safety the first priority' [3]. We would like to share our guidelines, in order to assist other departments in undertaking their own teleophthalmology service to provide safe and effective care.

Supplementary information The online version of this article (https:// doi.org/10.1038/s41433-020-01231-0) contains supplementary material, which is available to authorized users.

$\triangle$ Ji-Peng Olivia Li

o.li@nhs.net

1 Emergency Ophthalmology Clinical Research and Innovation Unit, Moorfields Eye Hospital NHS Foundation Trust, London, UK

2 NIHR Biomedical Research Centre for Ophthalmology, Moorfields Eye Hospital NHS Foundation Trust and UCL Institute of Ophthalmology, London, UK

3 UCL Institute of Ophthalmology, London, UK
Safe patient care must minimise missed or delayed diagnoses. Teleophthalmology cannot act as a replacement for a full ophthalmic examination, as currently slitlamp biomicroscopy is not possible via video-consultation platforms, and anterior segment examination relies on the quality of the video equipment used. Data protection contributes to a safe and effective video-consultation platform so our calls are encrypted, and no patient information is stored. It is possible to mitigate the limitations of teleophthalmology, for example by forming consultation guidelines for all clinicians staffing the service (Appendix 1). These include questions that must be asked in the history, particularly the presence or absence of 'red flag' symptoms such as sudden loss of vision or a patient who has recently had an invasive ophthalmic procedure. Such patients should be directed for a local timely face-toface consultation unless there are good clinical grounds not to do so. The second factor in reducing delayed or missed diagnoses is the use of 'safety net' advice at the end of the consultation. All patients should be advised of the need to seek a further review, often face-to-face, should any 'red flag' symptoms develop or should their condition worsen despite the treatment initiated at their teleophthalmology consultation.

The second GMC key principle for remote consultations is to 'identify vulnerable patients and take steps to protect them' [3]. Teleophthalmology services should ensure that patients unwilling or unable to travel, do not receive substandard care. Safe ophthalmology services must be inclusive. For those unable to undertake video-consultation, usually due to poor internet connectivity, or limited digital literacy, providers should ensure that other access routes, such as telephone helplines, Braille telephone helplines and walk-in emergency departments remain operational.

Accessibility is the key to safe consultations; patients will come to harm if they cannot access timely and effective healthcare. We chose to use the 'Attend Anywhere' (Attend Anywhere, Melbourne, Australia) video-consultation 
platform, as it requires only an internet connection and a video enabled device. The service is drop-in: no prior consultation with a general practitioner or optometrist is required, which facilitates efficient review and minimises barriers to access.

Any safe service has to be appropriately staffed. We recommend designing a service with appropriate administrative support, a suitable number of clinical staff for the patient workload and senior staff available for consultation. In our process, patients are initially consulted by a member of the administration team to ensure that their details are recorded correctly on the patient administration system and these are available to clinicians. During working hours, our staffing guidelines are that a consultant ophthalmologist oversees all sessions with residents, fellows and optometrists making up the remainder of the team with a predicted ratio of 6-8 consultations per team member per session. Out of hours an ophthalmology resident with 5 or more years' experience leads the team, and contact details for the clinician in charge and subspecialty teams are circulated at the start of each day. Communication between team members is via the secure Pando Messaging Application (Forward Clinical, London, UK) which is NHS approved for the transfer of patient identifiable information. We recommend that when a new service is established, there is sufficient administrative and senior clinical staffing support to reduce the risk of avoidable harm to patients and that there is accessible support for all team members.

GMC key principles 6 and 9 for remote consultations recommend that clinicians can 'access medical records' and 'keep notes that fully justify the decisions they make' [3]. This can pose a challenge in teleophthalmology as, for a number of reasons, consultations may take place away from hospital premises. Moorfields has been proactive in ensuring that all clinicians who require remote access have encrypted access via two factor authentication to the hospital electronic medical record. This ensures that previous records are to hand during video consultations to guide management decisions and allows clinicians to record their consultation and outcome directly into the electronic record. A letter proforma has been produced (Appendix 2) to ensure that communication is standardised across clinicians and that the key information is communicated after the consultation. Teleophthalmology services should be supported with good quality electronic health records, secure remote access and tools for standardised, contemporaneous documentation.
Evaluation of outcomes and reviewing practice is a further requirement of patient safety. Incidents arising from video consultations should be reported as for face-to-face consultations and units should collect user feedback. The authors would urge all providers of teleophthalmology to have robust monitoring processes in place for the service and be responsive to patient and user feedback.

In summary, we believe that teleophthalmology consultations are a safe way of assessing and treating patients with emergency ophthalmic conditions. A service can be adherent to the principles of remote consultations and prescribing as laid down by the GMC in the UK [3]. We hope that others can utilise our protocols and guidelines to assist in the design and running of their own teleophthalmology emergency service.

\section{Compliance with ethical standards}

Conflict of interest The authors declare that they have no conflict of interest.

Publisher's note Springer Nature remains neutral with regard to jurisdictional claims in published maps and institutional affiliations.

\section{Appendix 1}

Guidelines for clinicians undertaking emergency teleophthalmology consultations at Moorfields Eye Hospital.

\section{Appendix 2}

Proforma for use by clinicians following emergency teleophthalmology consultations for communication to patients and GP's.

\section{References}

1. Kang S, Thomas P, Sim D, Parker R, Daniel C, Uddin J. Oculoplastic video-based telemedicine consultations: Covid-19 and beyond. Eye. 2020;12:1-3.

2. Bourdon H, Jaillant R, Ballino A, El Kaim P, Debillon L, Bodin S, et al. Teleconsultation in primary ophthalmic emergencies during the COVID-19 lockdown in Paris: experience with 500 patients in March and April 2020. J Fr Ophtalmol. 2020;43:577-85.

3. General Medical Council. https://www.gmc-uk.org/ethical-guida nce/learning-materials/remote-prescribing-high-level-principles. Accessed Aug 2020. 